\title{
Antimicrobial Resistance among Uropathogenic Bacteria in Rural Kerala, India
}

\author{
T. Shailaja Sukumaran and A. Mohan Kumar* \\ Department of Microbiology, PK Das Institute of Medical Sciences, Vaniyamkulam, \\ Palakkad, Kerala, India \\ *Corresponding author
}

\section{A B S T R A C T}

Urinary tract infections caused by multidrug resistant bacteria are increasingly becoming prevalent even in rural areas. Objective of the present study was to find out the distribution

\begin{tabular}{|l|}
\hline Ke y w or d s \\
Urinary isolates, \\
Antibiotic \\
resistance, Rural \\
India. \\
\hline Article Info \\
\hline Accepted: \\
23 July 2017 \\
Available Online: \\
10 September 2017 \\
\hline
\end{tabular}
and antibiotic resistance pattern of urinary isolates over a period of one year in a tertiary care centre of Central Kerala which has got a rural location. We analyzed the types and antibiotic resistance pattern of all significant urinary isolates in the microbiology department from May 2016 - April 2017. Of the 3772 urine samples processed, $1265(33.5 \%)$ showed significant bacteriuria. The predominant isolate was E. coli (48.9\%) followed by Klebsiella sp. (14\%), Enterococcus sp. (14\%), Staphylococcus sp. (7\%), Pseudomonas and other non-fermenters (11\%). Among Enterobacteriaceae, 64.7\% was ESBL producers and $15.75 \%$ were carbapenem resistant. Around $63 \%$ of E.coli and Klebsiella sp. were found to be resistant to fluoroquinolones like norfloxacin and ciprofloxacin. Ampicillin resistance was observed in 35\% of Enterococcus isolates and vancomycin resistance in $0.65 \%$. Of the Staphylococcal isolates, $79.5 \%$ was methicillin resistant. From our study, we could realize that the problem of antibiotic resistance is increasing in an alarming rate even in rural areas. Multidrug resistance, since being a universal problem, we all have to be vigilant against this menace and the need for antibiotic stewardship should be reiterated.

\section{Introduction}

Urinary tract infection (UTI) is the most common of infections in the clinical practice. Among the bacterial agents of UTI, E. coli continues to be the commonest in both community acquired and hospital acquired cases (Gupta et al., 2002). Other pathogens include Klebsiella, Pseudomonas and other non-fermenters, Enterococcus, Staphylococcus, Enterobacter, Citrobacter, Proteus, etc. Over the last decade there has been an alarming increase in antimicrobial resistance (AMR) among the common bacterial agents of UTI in developing and developed countries. In this context, the empirical antibiotics used earlier in UTI such as ampicillin/amoxicillin, co-trimoxazole, norfloxacin, ciprofloxacin, etc. can no longer be used confidently. In the recent years antibiotic resistance is becoming a major problem not only in hospital acquired complicated UTIs but also in uncomplicated community acquired cases (Kavya et al., 2016) which makes the treatment of UTI more complex. On reviewing the literature it 
could be easily understood that AMR is not distributed uniformly among different countries and in different parts of the same country. Hence it is mandatory to establish routine mechanisms to assess local resistance rates among UTI pathogens. The standard regimen for empiric therapy should be reassessed periodically in light of changing susceptibility patterns. In the present study, we aim to find out the antibiotic resistance pattern of urinary isolates from a tertiary care centre in central Kerala against the commonly used drugs. An attempt is also made to find out the difference in resistance pattern among hospitalized and outpatient cases.

\section{Materials and Methods}

We conducted a prospective analysis of all significant urinary isolates in the microbiology department over a one year period from May, 2016 - April, 2017. Samples from clinically suspected cases of UTI, both hospitalized and out patients, irrespective of their age and sex from PK Das Institute of Medical Sciences, Palakkad, Kerala, India are included in the study. Most of the samples are clean catch mid-stream samples and the rest, catheter samples and supra pubic aspirates. All the samples are processed within $2 \mathrm{hrs}$ of collection. Microscopic examination and culture on MacConkey agar and 5\% sheep blood agar are done. Significance of the isolates is assessed by following the recommendations of Kass, microscopic findings as well as the clinical features. Colony counts $<10^{5}$ also are considered significant on an individual basis by correlating the microscopic findings, type of specimen and the clinical history.

Identification of the isolates is done by standard bacteriological methods (Collee et al., 2006). In addition, Hi Media, Hi Chrome UTI Agar is used for confirming the identification of Enterobacteriaceae and
Pseudomonas. Antibiotic susceptibility testing of the isolates is performed by KirbyBauer disc diffusion method on Mueller Hinton Agar as per CLSI guidelines (CLSI, 2016) using Hi Media, India antibiotic discs. Antibiotic discs used are ampicillin $(10 \mu \mathrm{g})$, norfloxacin $(10 \mu \mathrm{g})$, ciprofloxacin $(5 \mu \mathrm{g})$, nitrofurantoin $(300 \mu \mathrm{g})$, co-trimoxazole $(1.25 / 23.75 \mu \mathrm{g}), \quad$ cephalothin $\quad(30 \mu \mathrm{g})$, cefuroxime $(30 \mu \mathrm{g})$, cefotaxime $(30 \mu \mathrm{g})$, ceftazidime $(30 \mu \mathrm{g})$, gentamicin $(10 \mu \mathrm{g})$, piperacillin $(100 \mu \mathrm{g})$, amikacin $(30 \mu \mathrm{g})$, ampsulbactam $(10 / 10 \mu \mathrm{g})$, piperacillin-tazobactam $(100 / 10 \mu \mathrm{g})$, imipenem $(10 \mu \mathrm{g})$, meropenem $(10 \mu \mathrm{g})$, high level gentamicin $(120 \mu \mathrm{g})$, tetracycline $(30 \mu \mathrm{g})$, cefoxitin $(30 \mu \mathrm{g})$, vancomycin $(30 \mu \mathrm{g})$, linezolid $(30 \mu \mathrm{g})$.

Using CLSI phenotypic confirmatory test, ESBL production is checked with cefotaxime clavulanate disc among the members of Enterobacteriaceae. Quality control is performed using standard strains, E. coli ATCC 25922, Pseudomonas aeruginosa ATCC 27853, and Staphylococcus aureus ATCC 25923.

Considering the differences in the susceptibility pattern of bacterial agents of UTI among community acquired and hospital acquired cases, characterization of the isolates and their antibiotic resistance pattern based on the hospital status of the patients is also done.

\section{Results and Discussion}

Among the 3772 urine samples processed during the one year study period $1265(33.5 \%)$ samples gave significant bacterial growth.

The predominant isolate was E. coli $(48.9 \%)$ followed by Klebsiella sp. (14\%), Enterococcus sp. (14\%), Staphylococcus sp. (7\%), Pseudomonas and other non-fermenters (11\%) (Fig. 1). 61.7\% of total isolates in the present study is from inpatients and the rest is 
from out patients. In both the groups, E. coli stands as the commonest isolate, which constitutes $57.7 \%$ of the outpatient and $43.5 \%$ of inpatient isolates respectively. Members of enterobacteriaceae constitute $81.6 \%$ of the total isolations in outpatients, whereas in inpatients, it is only $59.8 \%$ (Fig. 2).

Among the members of Enterobacteriaceae isolated, $64.7 \%$ are ESBL producers and $15.75 \%$ were carbapenem resistant (Table 1).

Ampicillin resistance is observed in $35 \%$ of Enterococcus isolates and vancomycin resistance in $0.65 \%$. Of the Staphylococcal isolates, $79.5 \%$ is methicillin resistant (Table 2).

$58.9 \%$ of E. coli isolates from outpatients and $73.5 \%$ from admitted patients are tested positive for ESBL production. For all common organisms from both outpatients and hospitalized patients, nitrofurantoin and amikacin are found to be least resistant followed by piperacillin- tazobactam and carbapenems. For E. coli, nitrofurantoin resistance is $14.6 \%$ and $20.4 \%$ respectively, in outpatients and inpatients and for amikacin it is $2.9 \%$ and $11.8 \%$ (Table 3 ).

There are an estimated 150 million cases of UTIs per annum worldwide (Stamm et al., 2001) which along with the consistently increasing antibiotic resistance among the common causative agents of UTI makes the regular surveillance of the changing trends in antibiotic resistance pattern mandatory.

Even though our most common isolate is $E$. coli (49\%), the rate is less compared to western studies where the corresponding rate is about 85\% (George et al., 2000 and Richard Colgan et al., 2008). Data from other studies from India also show a similar reduced rate of 50-60\% (Mohammed Akram et al., 2007 and Dnyaneshwari et al., 2016) which is an important point to be considered. Another significant finding noticed in the present study, similar to studies from other parts of India (Mohammed Akram et al., 2007), is the rate of isolation of Klebsiella(14\%), which is more in this part of the world compared to US and Europe where the rate comes only to $3.8 \%$ (George et al., 2000). Along with Klebsiella, we have noted the emergence of Enterococcus also as a significant pathogen (14\%) which agrees with the findings of another recent study from Pune, India (Dnyaneshwari et al., 2016). However, in a study from Mysore, India the rate of isolation of Enterococcus was only $5.97 \%$ (Kavya et al., 2016). Rate of Pseudomonas and other NFGNBs is also increasing in this region and the same has also been reported by Dnyaneshwari et al., (2016). From the above observations we can infer that there is a region wise difference in the bacterial agents of UTIs.

Among the hospitalized patients, predominance of Enterococci, Pseudomonas, other NFGNB and Staphylococci is noted more than outpatients which corresponds with other studies (George et al., 2000 and Jharna Mandal et al., 2012). Co-trimoxazole, ampicillin, ciprofloxacin, early cephalosporins and nitrofurantoin were considered as the front line agents of treatment for UTIs all over the world. But recent regional and nationwide studies in US have reported that resistance to cotrimoxazole among urinary isolates of $E$. coli is approaching or exceeded $20 \%$ (James et al., 2006 and Gupta et al., 2001). In the present study, $58.8 \%$ of E. coli isolates are found to be resistant to co-trimoxazole and the mean resistance rate of all bacteria to cotrimoxazole is $57.1 \%$. The corresponding rate reported by Karlowsky et al., is $66.5 \%$ (James et al., 2006). In a study from Aligarh, India and according to Gupta et al., the rates are $60.4 \%$ and $80 \%$ respectively (Mohammed 
Akram et al., 2007 and Gupta et al., 2002). According to US Infectious Disease Society of America, co-trimoxazole cannot be considered as the empirical therapy for UTI where the resistance rate is $\geq 20 \%$ (James $e t$ al., 2006). Hence co-trimoxazole, no longer holds good as the first line agent of treatment in our area.

We could demonstrate a resistance rate of $91.1 \%$ for E. coli against ampicillin and a mean resistance rate of $84.2 \%$ for all the isolates together. Klebsiella sp. shows a mean resistance of $99.4 \%$ which could be explained by the inherent resistance of Klebsiella to ampicillin. Many other reports from India also show a high resistance of about $80-98 \%$ against Klebsiella and E. coli, (Dnyaneshwari et al., 2016 and Jharna Mandal et al., 2012). In most parts of the world, the rate of ampicillin resistance among urinary isolates is increasing, which according to a study from North America in 2006 is $79.8 \%$ (James et al., 2006) and this shows that ampicillin has no bearing in the empirical therapy of UTIs.

Considering the high rate of resistance to cotrimoxazole and ampicillin, in many parts of US and Europe, quinolones have been used as the main drugs for UTI (George et al., 2000 and James et al., 2006). However, following its widespread use, studies from various parts of US and Spain report that fluoroquinolones demonstrate a stepwise, threefold increase in resistance from $1995(0.7 \%)$ to $2001(2.5 \%)$ (James et al., 2002). In the present study, mean resistance of all isolates to ciprofloxacin is $51 \%$ in comparison to $18 \%$ from a Spanish study (Gobernado et al., 2007). Other Indian studies also have reported a similar high level resistance (Jharna Mandal et al., 2012 and Mohammed Akram et al., 2007).

Fig.1 Distribution of the isolates

\section{Distribution of Isolates}

PERCENTAGE

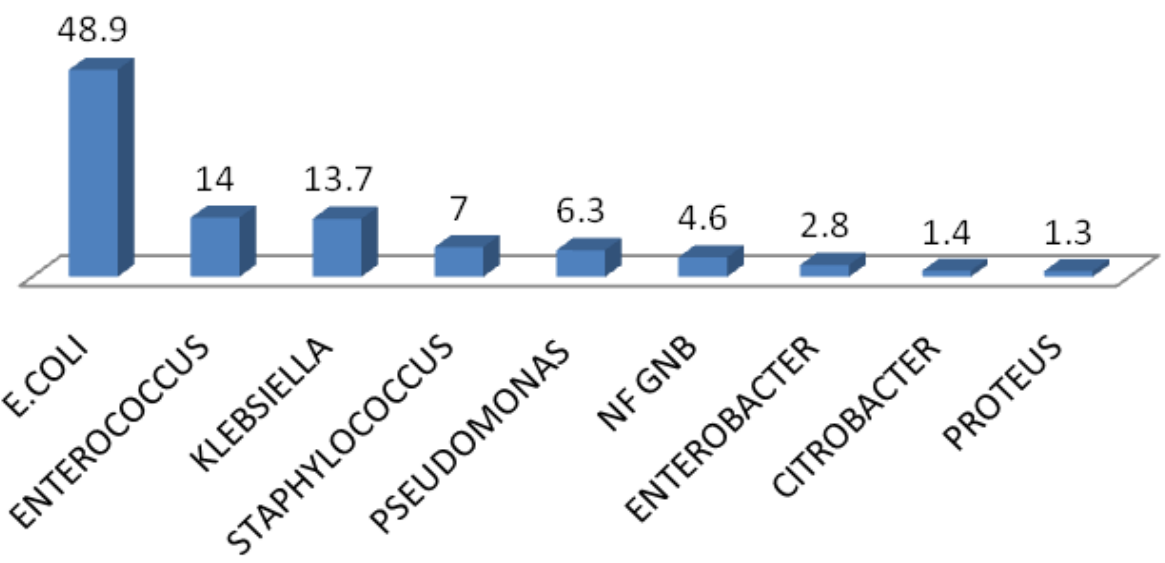


Fig.2 Distribution of isolates based on hospital status of the patients

\section{Distribution of isolates based on hospital status of the patients}

$\square \mathrm{OP}(\%) \quad \square \mathrm{IP}(\%)$

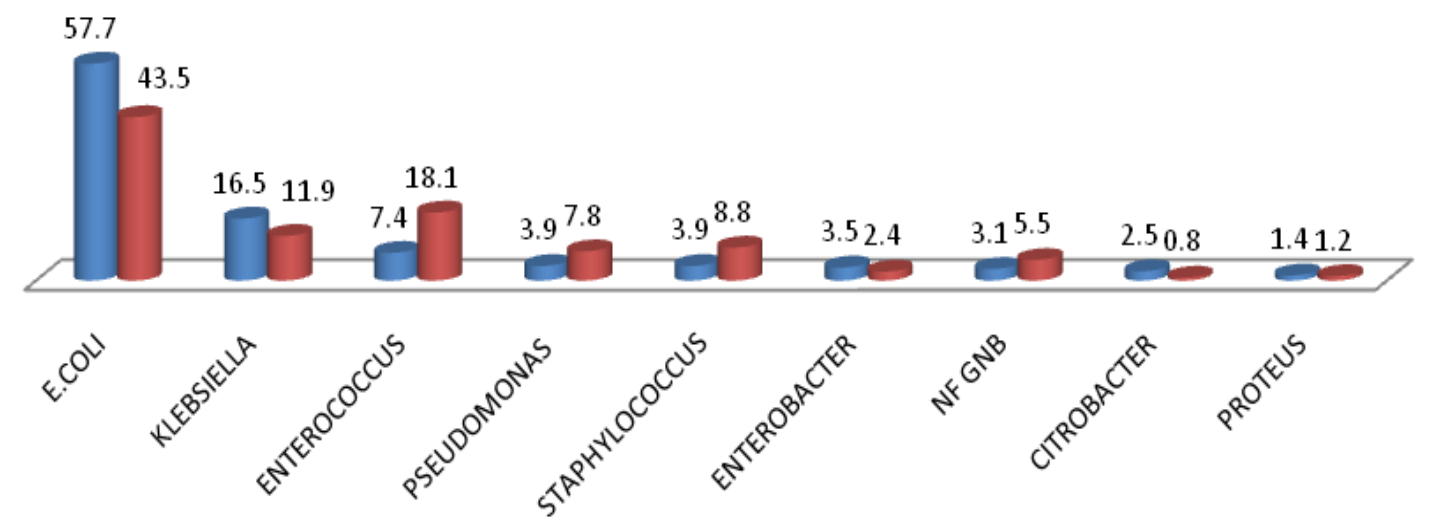

Table.1 Antibiotic resistance pattern of the Gram negative isolates

\begin{tabular}{|c|c|c|c|c|c|c|c|}
\hline & $\begin{array}{c}\begin{array}{c}\text { E.coli } \\
(\mathbf{n}=619) \\
\%\end{array} \\
\text { \% }\end{array}$ & $\begin{array}{c}\text { Klebsiella } \\
\text { sp } \\
(\mathrm{n}=173) \\
\%\end{array}$ & $\begin{array}{c}\text { Proteus } \\
\text { sp } \\
(\mathrm{n}=16) \\
\%\end{array}$ & $\begin{array}{c}\text { Citrobacter } \\
s p \\
(\mathrm{n}=18) \\
\%\end{array}$ & $\begin{array}{c}\text { Pseudomonas } \\
\text { sp } \\
(\mathrm{n}=\mathbf{8 0}) \\
\%\end{array}$ & $\begin{array}{c}\text { Other } \\
\text { NFGNB } \\
(\mathbf{n}=58) \\
\%\end{array}$ & $\begin{array}{c}\text { Enterobacter } \\
s p \\
(\mathrm{n}=36) \\
\%\end{array}$ \\
\hline AMP & 91.1 & 99.4 & 56.1 & 100 & 80 & 100 & 100 \\
\hline CEP & 83.7 & 82.1 & 56.1 & 27.8 & 78.8 & 100 & 97.2 \\
\hline CXM & 72.7 & 70.5 & 50 & 22.2 & 78.8 & 100 & 75 \\
\hline CTX & 70.1 & 67.1 & 12.5 & 22.2 & 52.5 & 87.9 & 63.9 \\
\hline CAZ & 70.3 & 67.1 & 12.5 & 22.2 & 52.5 & 74.1 & 63.9 \\
\hline COT & 58.8 & 64.2 & 25 & 16.7 & 100 & 46.1 & 58.3 \\
\hline CIP & 66.4 & 59.5 & 12.5 & 16.7 & 52.5 & 60.3 & 38.9 \\
\hline NX & 66.7 & 59.5 & 18.8 & 16.7 & 53.8 & 60.3 & 36.1 \\
\hline NIT & 17.8 & 75.1 & ND & 61.1 & 77.5 & 93.1 & 88.9 \\
\hline $\mathrm{AK}$ & 7.8 & 26 & 0 & 16.7 & 40 & 63.8 & 33.3 \\
\hline GEN & 35.1 & 49.1 & 0 & 16.7 & 47.5 & 62.1 & 52.8 \\
\hline PI & 37.4 & 82.1 & 18.8 & 27.8 & 40 & 75.9 & 72.2 \\
\hline PIT & 25.5 & 45.1 & 0 & 16.7 & 33.8 & 48.3 & 47.2 \\
\hline $\mathrm{A} / \mathrm{S}$ & 75 & 83.2 & 25 & 50 & 80 & 55.2 & 88.8 \\
\hline IPM & 7.9 & 17.3 & 6.3 & 5.6 & 25 & 50 & 22.2 \\
\hline MRP & 20.4 & 27.7 & 0 & 5.6 & 46.3 & 41.4 & 22.2 \\
\hline ESBL & 66.9 & 67.1 & 6.3 & 22.2 & $\mathrm{ND}$ & ND & 63.9 \\
\hline
\end{tabular}

Amp-ampicillin, Cep-cephalothin, Cxm-cefuroxime, Ctx-cefotaxime, Caz-ceftazidime, Cip-ciprofloxacin, Gengentamicin, Ak-amikacin, Cot-co-trimoxazole, Nx-norfloxacin, Nit-nitrofurantoin, A/s-amp- sulbactam, PI piperacillin, PIT-piperacillin-tazobactam, Ipm- imipenem, Mrp-meropenem, ND-not done 
Table.2 Antibiotic resistance pattern of the Gram positive isolates

\begin{tabular}{|c|c|c|}
\hline & $\begin{array}{c}\text { Enterococcus } \boldsymbol{s} \boldsymbol{p} \\
(\mathbf{n = 1 7 7 )} \mathbf{\%}\end{array}$ & $\begin{array}{c}\text { Staphylococcus } \boldsymbol{s} \boldsymbol{p} \\
(\mathbf{n = 8 8}) \mathbf{\%}\end{array}$ \\
\hline AMP & 35 & 96.6 \\
\hline COT & 80.2 & 44.3 \\
\hline CIP & 78 & 73.9 \\
\hline NX & 77.4 & 73.9 \\
\hline NIT & 18.1 & 0 \\
\hline AK & 59.3 & 71.6 \\
\hline GEN & 55.9 & 55.7 \\
\hline PI & 35 & 90.9 \\
\hline PIT & 35 & 81.8 \\
\hline A/S & 52 & 83 \\
\hline VA & 0.6 & 0 \\
\hline LZ & 0 & 0 \\
\hline CX & ND & 79.5 \\
\hline
\end{tabular}

Amp-ampicillin, Cip-ciprofloxacin, Gen-gentamicin, Ak-amikacin, Cot-co-trimoxazole, Nx-norfloxacin, Nitnitrofurantoin, A/s-amp- sulbactam, PI - piperacillin, Pit-piperacillin-tazobactam, Cx - cefoxitin, Va-vancomycin, Lz-linezolid, ND-not done.

Table.3 Distribution of antibiotic resistance based on patients hospital status

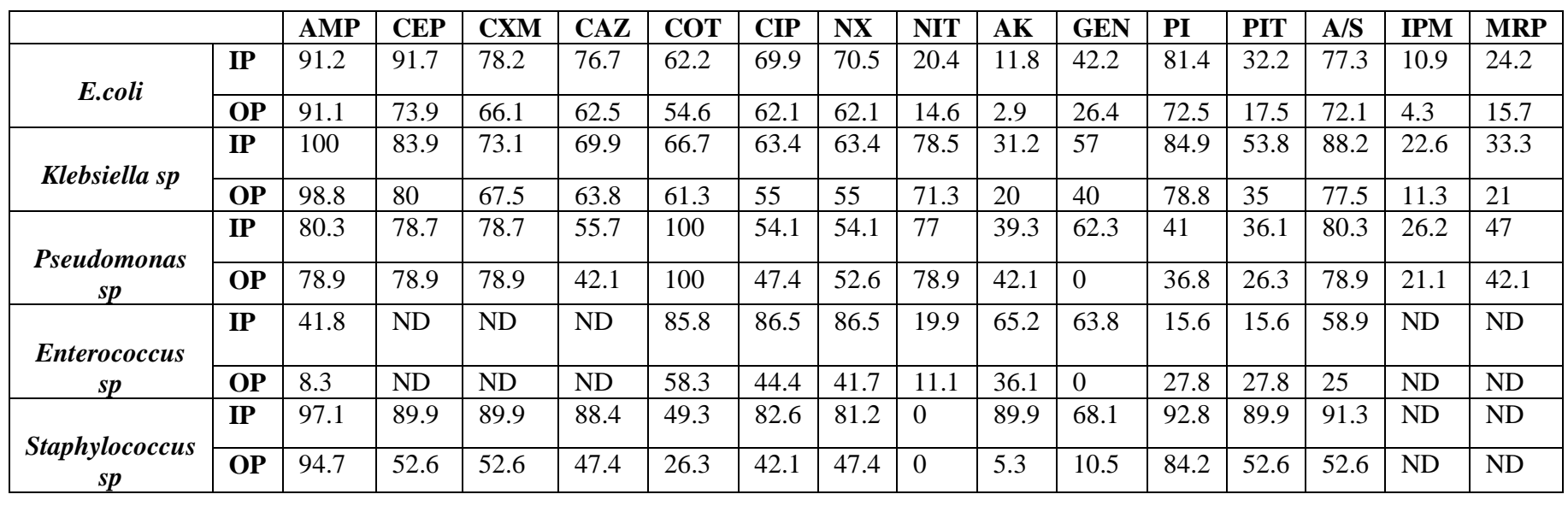

Nitrofurantoin which still remains as an important treatment option for UTI in western countries (George et al., 2000) has a mean resistance of $59 \%$ against the urinary pathogens of our study. The resistance rate varies among different pathogens, for $E$. coli it is $17.8 \%$, Klebsiella $75.1 \%$, for Enterococcus and Staphylococcus $18.1 \%$ and $0 \%$ respectively. In various studies from US, the mean rate of nitrofurantoin resistance for urinary isolates is around $4.5 \%$ (James et al., 2006 and George et al., 2000) compared to $34.3 \%$ from a multicenter study in Delhi (Atul et al., 2008). Regardless of the wide spread use for a long period, this drug maintains a low level of resistance which could be explained by its exclusive pharmacodynamic properties (McOsker et al., 1994). Since adequate concentration of this drug cannot be obtained in blood, nitrofurantoin can be used only in lower UTIs.

All the isolates from the present study show a substantially high level of resistance to most of the second line antibiotics; amikacin, extended spectrum cephalosporins, inhibitor 
combinations like ampicillin- sulbactam and piperacillin-tazobactam. In a study from Goa, India, $73.5 \%$ of the etiological agents of hospital acquired UTI were resistant to all the antibiotics tested (Umesh S Kamat et al., 2009). In another study from Aligarh, India, resistance of $E$. coli to third generation cephalosporins was 55-85\% (Mohammed Akram et al., 2007). Similarly in the present study $64.7 \%$ of Enterobacteriaceae are ESBL producers and $37 \%$ of the isolates are resistant to piperacillin tazobactam. We could demonstrate a mean resistance of $35.4 \%$ to aminoglycoside, amikacin and $41.7 \%$ for gentamicin. Mean resistance to imipenem in Gram negative isolates is $19.2 \%$ and the same for meropenem is $23.4 \%$. This slight increase in resistance against meropenem compared to imipenem noted among our isolates could be due to the increased use of the former in clinical practice. In a study among community acquired UTI cases by Atul et al., meropenem resistance for E.coli was $0 \%$ whereas it is $15.7 \%$ in the present study (Atul et al., 2008). In another study from Gujarat, carbapenem showed only $16 \%$ sensitivity in nosocomial UTI and $41.3 \%$ sensitivity in communityacquired UTI and colistin had a total sensitivity of $45.3 \%$ only (Raval et al., 2015). However no colistin resistance is detected among our isolates.

Enterococcus sp. is a significant isolate in this study which constitutes $14 \%$ of total isolates. These strains exhibit a mean resistance of $35 \%$ to ampicillin and the rate of vancomycin resistant Enterococcus comes to $0.6 \%$. No case of vancomycin resistant Staphylococcus is isolated and the rate of methicillin resistance is $79.5 \%$ in the present study. Pseudomonas isolates are highly resistant to all the antimicrobials tested except imipenem (25\%) and piperacillin tazobactam (33\%). Other NFGNB exhibit a reasonably high resistance to all the antibiotics except colistin. Many recent studies also have reported increasing rates of carbapenem resistant Acinetobacter isolates (Li-Yang $\mathrm{Hsu}$ et al., 2017 and Paul G. Higgins et al., 2010).

Among the outpatients, $39.9 \%$ of E.coli isolates are ESBL producers whereas it is almost double in admitted patients. This shows the increased rate of AMR in hospitalized patients compared to community acquired cases as reported in similar studies from India (Kavya et al., 2016 and Atul et al., 2008). 87\% of Staphylococcal strains from admitted and $52.6 \%$ from outpatients are methicillin resistant which shows the increased prevalence of community acquired methicillin resistance in our locality. In our study, ciprofloxacin sensitivity to E.coli among outpatients is $37.9 \%$ whereas it was only $28 \%$ in a study from Delhi (Atul et al., 2008).

Nitrofurantoin and amikacin are found to be least resistant among the common organisms in both outpatients and hospitalized patients. Even though aminoglycosides have been around for a long while, resistance has not developed as rapidly as to others. The chief reason for this appears to be that these drugs being administered parenterally have not been over-used. In E. coli, nitrofurantoin resistance is $14.6 \%$ and $20.4 \%$ respectively in outpatients and admitted patients which makes this as the first line of treatment in all cases of UTI.

Multidrug resistance (MDR) is very common among uropathogens as reported by Akram et al., where almost all isolates were MDR (Mohammed Akram et al., 2007). In a large scale study on 38,835 urinary isolates of $E$. coli, only $7.1 \%$ were MDR (Daniel et al., 2001) whereas $65.9 \%$ of our E. coli isolates are resistant to three or more groups of the first line drugs which is incomparably higher than western experience. Indian isolates show higher resistance than the isolates from USA 
and Europe. The possible explanation is the uncontrolled antibiotic prescribing practices of our region. Another reason for high rate of resistance in the present study is that the isolates which are tested in a laboratory based study may be mostly from previous antibiotic treatment failed or from patients with other underlying risk factors. Hence this study, like any other laboratory based study, may bias towards an over reporting of resistance in patients with UTI and necessitates more patient based studies in this area.

All over the world, the problem of antibiotic resistance is increasing in a fast pace. Various studies on antibiotic resistance among uropathogens around 2000 show a low rate of resistance to ampicillin, co-trimoxazole, nitrofurantoin and quinolones in US and European countries (James et al., 2002, Daniel et al., 2001). In 2007, a large scale study conducted in Spain showed a drastic increase in the resistance to ampicillin, cotrimoxazole and quinolones, 52.1\%, 26\% and $18 \%$ respectively (Gobernado et al., 2007). There are several factors which contribute to this high level of antibiotic resistance in bacteria. One of the main reasons for $E$. coli resistance to quinolones in Spain has been ascribed to the over use of these drugs (Gobernado et al., 2007). Other factors like use of quinolones in agriculture and in animals, for growth promotion are also contributing (Garau et al., 1999). The above mentioned reasons must have contributed to the high rates of resistance among our isolates as well. More problem oriented, institutional, regional and nationwide studies are required in our country to understand the changing trends of antibiotic resistance among urinary isolates. The implementation of hospital antibiotic policies also will help to check the uncontrolled increase in bacterial drug resistance to a certain extent.

Being a tertiary care centre, most of our patients are referred cases and must have received various antibiotics from the peripheral practitioners which in turn must have helped in selecting out the resistant mutants. Use the appropriate antibiotic in correct dosage and duration, only in indicated cases. This should be our policy to tackle this growing menace.

Considering many studies from US, Europe and India, it is clear that antimicrobial resistance is markedly high in countries like India. Hence international treatment guidelines for empirical therapy in UTI will not stand true in Indian setting. This makes routine culture and sensitivity mandatory in all cases which is quite impractical also, to prevent treatment failure and development of further drug resistance. Therefore it is important to have periodic region wise analysis of changing trends of drug resistance among urinary isolates to formulate local guidelines for treating UTI. We hope that this study would help the clinicians in deciding the antibiotic therapy for UTIs and also would help in formulating an antibiotic policy in the hospitals.

\section{References}

Atul Kothari, Vishal Sagar. Antibiotic resistance in pathogens causing community-acquired urinary tract infections in India: a multicenter study, J Infect Developing Countries 2008; 2(5): 354-58.

Clinical and Laboratory Standards Institute. Performance Standards for Antimicrobial Susceptibility Testing. $26^{\text {th }}$ ed. CLSI Supplement M100S. Wayne, PA: Clinical and Laboratory Standards Institute; 2016.

Collee, J.G., Fraser AG, Marmion BP, Mackey SA, McCartney. Practical Medical Microbiology. In: Collee JG, Miles RS, Watt B, editors. Tests for the identification of Bacteria. 14th Ed. New Delhi, India: Elsevier; 2006. p. 131-49. 
Daniel F. Sahm, Clyde Thornsberry David C. Mayfield, Mark E. Jones, and James A. Karlowsky. Multidrug-Resistant Urinary Tract Isolates of Escherichia coli: Prevalence and Patient Demographics in the United States in 2000. Antimicrob Agents Chemother. 2001; 45(5): 1402-1406.

Dnyaneshwari Puroshottam Ghadage, Vrishali Avinash Muley, Jyotika Sharma, Arvind Vamanrao Bhore. Bacteriological Profile and Antibiogram of Urinary Tract Infections at a Tertiary Care Hospital. National Journal of Lab Med. 2016; 5(4): MO20-MO24.

Garau, J., Xercavins, M., RodríguezCarballeira, M. et al., Emergence and dissemination of quinolone-resistant Escherichia coli in the community. Antimicrob Agents Chemother 1999; 43: 2736-2741.

George G. Zhanel, James A. Karlowsky, Godfrey K. M. Harding, Anita Carrie, Tony Mazzulli, Donald E. Low, The Canadian Urinary Isolate Study Group and Daryl J. Hoban. A Canadian National Surveillance Study of Urinary Tract Isolates from Outpatients: Comparison of the Activities of Trimethoprim- Sulfamethoxazole, Ampicillin, Mecillinam, Nitrofurantoin, and Ciprofloxacin. Antimicrob Agents and Chemother. 2000; 44(4):1089-1092

Gobernado, M., Valdes L, Alos JI, GarciaRey C, Dal Re R, J Garcia -de-Lomas and the Spanish surveillance group for urinary pathogens. Antimicrobial susceptibility of clinical Escherichia coli isolates from uncomplicated cystitis in women over a1 year period in Spain. Rev. Esp. Quimioterap, Enero 2007; 20(1):68-76

Gupta, K., Daniel, David C. Mayfield, Stamm W.E. Antimicrobial resistance among uropathogens that cause community acquired UTI in women; a nationwide analysis. Clin.ID.2001; 33:89-94

Gupta, V., Yadav A, Josh RM. Antibiotic resistance pattern in uropathogens. IJMM. 2002; 20(2):96-98

James A. Karlowsky, Daryl J Hoban, Melanie $R$ De Corby, Nancy $M$ Laing and George G Zhanel. Flouroquinolone resistant urinary isolates of E. coli from outpatients are frequently multidrug resistant; Result from the North American collaborative alliance. Quinolone resistant study. Antimicrob Agents and Chemother. 2006; 50(6): 2251-2254

James A. Karlowsky, Laurie J. Kelly, Clyde Thornsberry, Mark E. Jones and Daniel F. Trends in Antimicrobial Resistance among Urinary Tract Infection isolates of Escherichia coli from Female Outpatients in the United States. Antimicrob agents and Chemother. 2002; 46(8):2540-2545

Jharna Mandal, SrinivasAcharya N, Buddhapriya D \& Subhash Chandra Parija. Antibiotic resistance pattern among common bacterial uropathogens with a special reference to ciprofloxacin resistant Escherichia coli. Indian J Med Res. 2012; 136(11): 842-49.

Kavya, S., Anuradha K. Comparison of bacteriological profile and susceptibility patterns to empiric antibiotics in hospital and community acquired urinary tract infection. IOSR Journal of Dental and Medical Sciences (IOSRJDMS). 2016; 15(3):05-09

Li-Yang Hsu, Anucha Apisarnthanarak, Erum Khan, Nuntra Suwantarat, Abdul Ghafur and Paul Anantharajah Tambyah. Carbapenem-Resistant Acinetobacter baumannii and Enterobacteriaceae in South and Southeast Asia. Clin. Microbiol. Rev. 2017; 30 (1): 1-221

McOsker, C.C., and Fitzpatrick PM, Nitrofurantoin mechanisms of action 
and implications for resistance development in common uropathogens. J. Antimicrob. Chemother. 1994 May; 33 Suppl A: 23-30

Mohammed Akram, Mohammed Shahid and Asad U Khan. Etiology and antibiotic resistance patterns of communityacquired urinary tract infections in $\mathrm{J} \mathrm{N}$ M C Hospital Aligarh, India. Ann Clin Microbiol Antimicrob.2007; 6:4.

Paul G. Higgins, Cathrin Dammhayn, Meredith Hackel, Harald Seifert. Global spread of carbapenem-resistant Acinetobacter baumannii. J Antimicrob Chemother.2010; 65 (2): 233-238.

Raval, R., Verma, R.J. and Kareliya H. Clinico-Pathological Features of Urinary Tract Infection in Rural India.
Advances in Infectious Diseases. 2015; 5: 132-39.

Richard Colgan, James R Johnson, Michael Kuskowski and Kalpana Gupta. Risk factors for Trimethoprim sulfamethoxazole resistance in patients with acute uncomplicated cystitis. Antimicrob agents and Chemother.2008; 52(3):846-851

Stamm, W.E., and SR Norrby. Urinary tract infections: disease panorama and challenges. $J$ Infect Dis. 2001; 183(suppl.1):S1-S4

Umesh S. Kamat, Agneto Fereirra, Dilip Amonkar et al., Epidemiology of hospital acquired urinary tract infections in a medical college hospital in Goa. Ind. J. Urol.2009; 25(1):76-80

\section{How to cite this article:}

Shailaja Sukumaran, T. and Mohan Kumar, A. 2017. Antimicrobial Resistance among Uropathogenic Bacteria in Rural Kerala, India. Int.J.Curr.Microbiol.App.Sci. 6(9): 2287-2296. doi: https://doi.org/10.20546/ijcmas.2017.609.280 